\title{
Control of Water Flow in Cooling of Thermal Power Plants
}

\author{
${ }^{1}$ Ayrat I. Badriev, ${ }^{2}$ Vilen N. Sharifullin, ${ }^{3}$ Andrew V. Sharifullin \\ ${ }^{1}$ Kazan Federal University \\ ${ }^{2}$ Kazan State Power Engineering University \\ ${ }^{3}$ Kazan National Research Technological University \\ Email: aibadriev@gmail.com
}

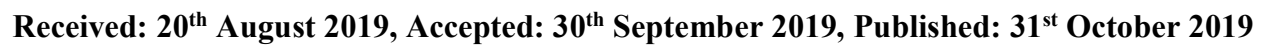

\begin{abstract}
The water flow rate of the cooling system of tower cooling towers is considered as a controlled parameter. Using data on the irregularity of irrigation density over the cross-section, a regression model of the operating characteristic of a tower cooling tower was obtained, which establishes the dependence of the temperature difference on the irrigation density. Based on this characteristic, the existence of an extremum of the cooling capacity of the cooling tower in terms of the flow rate of the flow is established. A mathematical analysis of the circulation flow control using shutoff valves in a single-circuit water supply system is carried out. The equation of the relationship between the flow rate of the circulating flow and the control position of the shutoff valves is given. An energy-saving method for controlling the performance of a circulation pump using frequency regulation is considered. A mathematical model of the hydrodynamics of the circulation circuit is constructed that describes the dependence of the circulation flow on the parameters of the shutoff valves and/or the frequency controller of the pump drive. A system for automatically controlling the vacuum in the condenser of a steam turbine plant is proposed. The circulation flow can serve as an optimizing and controlling parameter of the cooling capacity of the system. The developed mathematical model of a circulating system controlled by a frequency regulator of an electric drive of a circulation pump and/or shutoff valves of heat exchangers is recommended for use in solving the design and control problems of a circulating system. In automated process control systems, it can be used both for calculating the cooling capacity and unit energy consumption and in combination with regulation laws for flow control. This will improve the quality of the transition process, and, consequently, increase the efficiency and safety of production.
\end{abstract}

\section{Keywords}

Cooling System, Water Flow, Tower Cooling Tower, Shutoff Valves, Frequency Regulation.

\section{Introduction}

At industrial enterprises, among the systems of technical water supply, the system of reverse cooling of water with cooling towers has prevailed. Repeated use of circulating water can significantly reduce the consumption of fresh water in large quantities. At the same time, it is necessary that the temperature of the cooling water be as low as possible. In the energy production, the pressure in the condenser depends on the cooling temperature, which in turn determines the final parameters of the steam and, therefore, the efficiency of the turbine unit. In practice, the cooling ability, as the main characteristic of the cooling tower, has deviations and often does not reach optimal performance indicators. Ways to increase efficiency are given, for example, in [1-3], however, issues of controlling the flow of water along the circulation circuit are not considered. The work is devoted to the analysis of the influence of the circulation flow on the efficiency of the tower, as well as the mathematical analysis of the control of the circulation flow.

\section{Methods}

In a reverse cooling system with tower cooling towers of energy enterprises, it can be found that the only parameter that can be controlled is the flow of circulating water. During the operation of the system, as a control object, disturbing influences can influence, which could be made up with the help of the circulation water flow. The problem arises of analyzing the influence of the circulation flow on the efficiency of the process of cooling water in a tower. As a rule, when modeling and managing the system, it is necessary to know the experimental-calculation formulas. In particular, conducting an experiment to determine these formulas is difficult because it requires intervention in the process and decommissioning of equipment. Therefore, the article proposes a simplified method for assessing the performance of a tower-based on the irregularity of the density of irrigation in its sections. This solution was implemented using the example of a cooling tower with an irrigation area of $2600 \mathrm{~m}^{2}$. At an outdoor temperature of $25-31{ }^{0} \mathrm{C}$, since the summer, the differences of the temperatures $\Delta T$ and the irrigation density $q$ were measured at four different points in each section (12 sections in total) of the apparatus. According to the results, a significant irregularity of the irrigation density was obtained and methods for taking this unevenness into account were proposed [4]. Assume that the sections of the tower are identical to each other. Then, the experimental data can be combined to build the operating characteristic of the cooling tower (Fig. 1) 


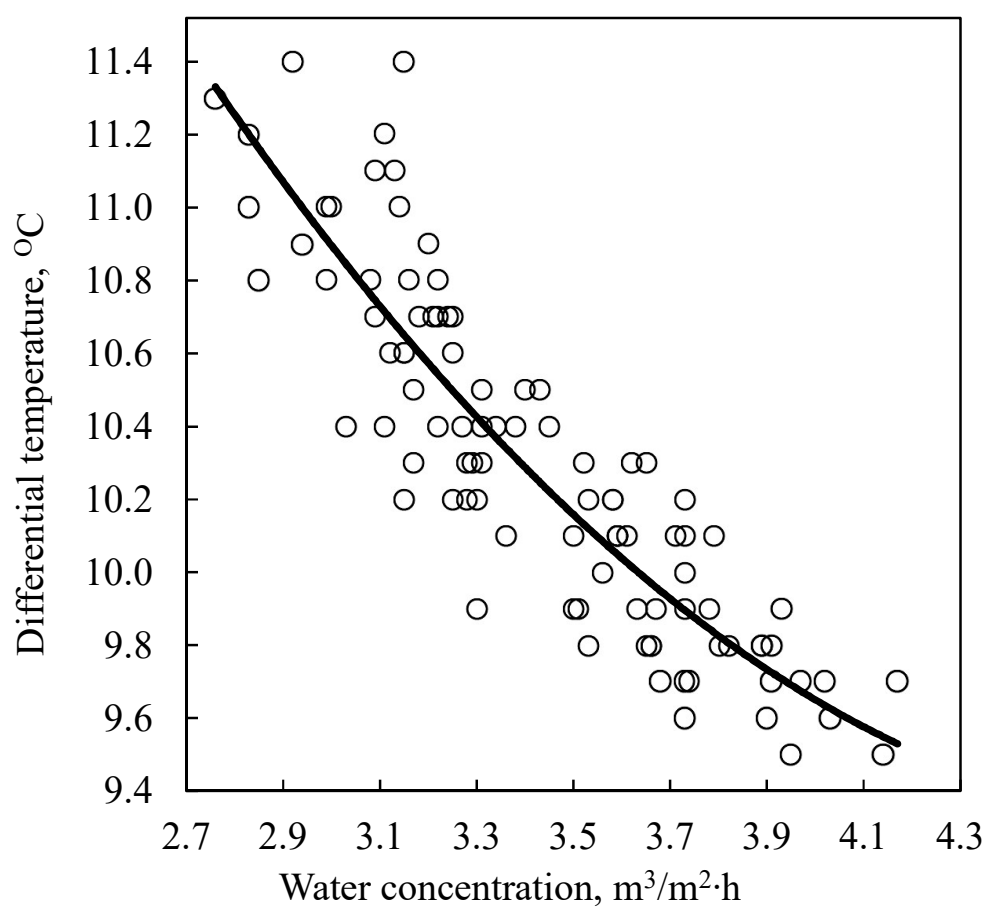

Figure 1: Performance Characteristics of the Effectiveness of a Cooling Tower Based on Irregularity of Irrigation Density

The obtained working characteristic is described by the parabolic type regression equation:

$\Delta T=23,3-4,46 \cdot q+0.279 \cdot q^{2}$

The cooling capacity of the tower can be calculated according to the formula:

$R=c_{p} \cdot \rho \cdot F \cdot q \cdot \Delta T$,

where $C_{p}$ is the specific heat of water, $\mathrm{kJ} /\left(\mathrm{kg} \cdot{ }^{\circ} \mathrm{C}\right) ; F$ - the cross-sectional area of the tower, $\mathrm{m}^{2} ; \rho$ - fluid density, $\mathrm{kg} /$ $\mathrm{m}^{3}$.

According to the obtained regression equation, a numerical analysis of the cooling power of the tower cooling tower was carried out. It was found that when varying the density of irrigation within the limits of workloads, the cooling power passes through a maximum value. It is entirely conceivable that the optimum value of cooling power for tower towers may be within, or beyond, the limits of workloads. Therefore, the circulating flow of the circulating system can act as an optimizing parameter of the system.

At the next stage, a method for regulating the circulating water along the circuit of the circulating system is considered. For clarity, Figure 2 shows a single-circuit cooling circuit, the main equipment of which is a cooling tower, circulating pump and condenser. Chilled water flows by gravity into the chamber, from where it is pumped out by a circulation pump and fed to the condenser. Heated water from the condenser enters the tower inlet, after which a new cooling cycle begins. The circulation pump develops a fairly powerful head and flow in the system circuit. According to the passport characteristics of the axial vertical pump OPV3-110 used in the revolving system, at the I speed the pump flow can be $14200 \mathrm{~m}^{3} / \mathrm{h}$, at II speed $-21600 \mathrm{~m}^{3} / \mathrm{h}$, with the corresponding pump operating capacities of 1000 and $1600 \mathrm{~kW}$. Poor regulation of such volumes of water flow leads to the low operational efficiency of the pump and high energy costs. So, the energy consumption for circulation pumps of TPPs can be about $23 \%$ of the total consumption for own needs [7]. 


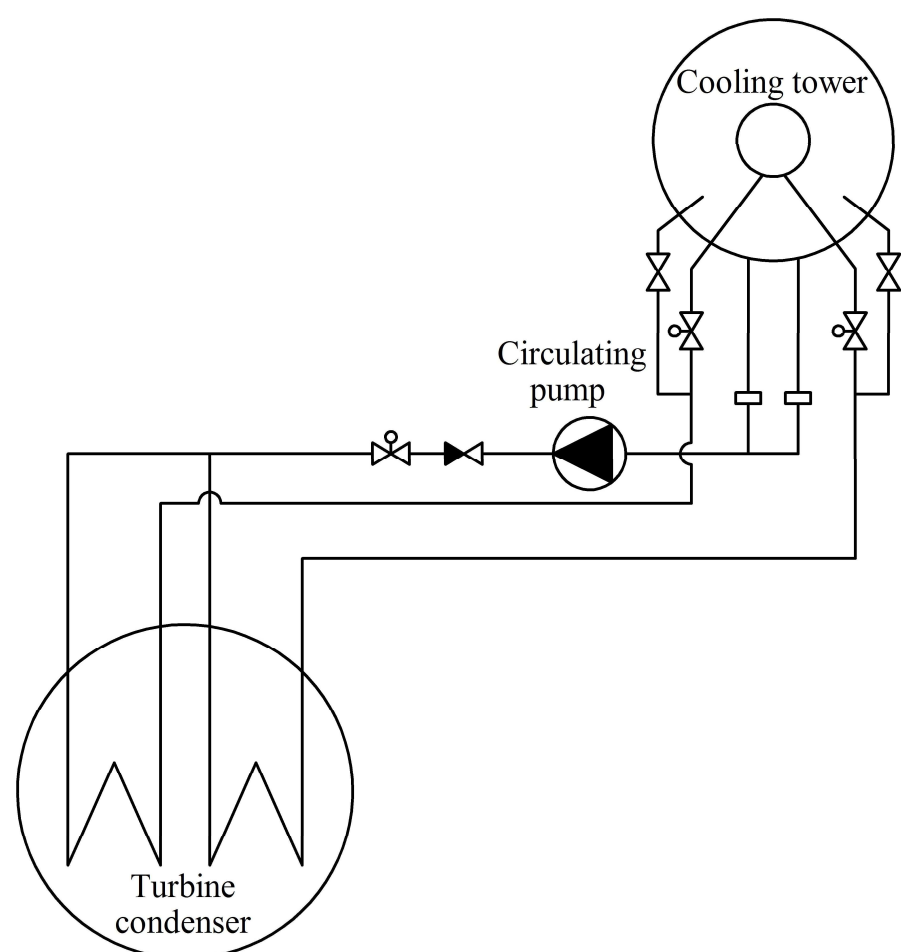

Figure 2: Single-Circuit Scheme for Reverse Cooling TPP

The fluid flow, using a circulation pump, as a rule, can be regulated in two ways: by throttling the fluid flow and by frequency regulation of the pump electric drive. Each of these methods has its own control parameter. When throttling, it is the position of the shutoff valve on the pump outlet pipe, with frequency regulation, the number of revolutions of the pump motor. When calculating the circulating system, it is important to understand how the control parameter and the circulation water flow are interconnected. Therefore, the mathematical relationship between the control parameter and the flow rate of the circulating flow during the throttling of the flow and frequency regulation of the motor speed is considered below.

We carry out a mathematical analysis of the control of water flow in a single-circuit circulating system during throttling. The pressure loss from the circulation pump to the cooling tower, with the resistance of the stop valves, can be calculated according to the Bernoulli equation according to the formula:

$$
H=\left[1+h+\lambda_{\mathrm{c}} \frac{L_{\mathrm{c}}}{D}+\lambda_{\mathrm{T}} \frac{L_{\mathrm{T}}}{D}+\sum \xi_{\mathrm{c}}+\xi_{\mathrm{a}}(y)\right] \frac{Q^{2} \rho}{2 S^{2}},
$$

where $H$ - pressure loss from the circulation pump to the cooling tower, $\mathrm{m}$; $h$ - the height of the rise of water in the cooling tower, $\mathrm{m} ; Q$ - volumetric flow rate of water, $\mathrm{m}^{3} / \mathrm{h} ; \lambda_{\mathrm{c}}$ and $\lambda_{\mathrm{T}}$ - coefficient of hydraulic resistance of the network of pipelines and condenser; $L_{\text {with }}$ - pipeline length, $\mathrm{m} ; L_{\mathrm{T}}$ - the length of the pipes of the capacitor; $\Sigma \xi_{\text {with }}$ - total coefficient of local resistance of pipelines and condenser; $\xi_{\text {a }}(y)$ - coefficient of resistance of the electromagnetic valve; $S$ - pipe crosssectional area, $\mathrm{m} ; D$ - pipeline diameter, $\mathrm{m}$; $d$ - diameter of condenser pipes, $\mathrm{m}$.

In a circulating system with shutoff valves, in particular, it is an electromagnetic valve, the pressure loss is quite large. The relative position of the regulatory body - the locking sleeve may vary within $0 \leq y \leq 1$. Then, with $y=0$, the resistance coefficient of the electromagnetic valve can take the boundary position $\xi_{a}(0)=\infty$, which corresponds to the "closed" position, and with $y=1$, the boundary position $\xi_{\mathrm{a}}(1)=\mathrm{min}$, which characterizes the open position. Given the variety of designs of valves, its resistance coefficient $\xi_{\mathrm{a}}(y)$ can be determined by a linear, parabolic or logarithmic dependence. The electromagnetic valve is characterized by a linear shape, and the dependence for the water flow will take the form [5]:

$\xi_{\mathrm{a}}(y)=\frac{2 g S^{2}}{\left(Q_{0} y\right)^{2}}$,

where $Q_{0}$ is the throughput of pipeline valves (with a pressure drop of $1 \mathrm{kgf} / \mathrm{cm}^{2}$ ), $\mathrm{m}^{3} / \mathrm{h}$.

The power $N$ and the pressure of the circulation pump $H$ can be calculated by the formulas: 


$$
\begin{aligned}
& \xi_{\mathrm{a}}(y)=\frac{\rho g H Q}{3600 \cdot 1000 \eta} ; \\
& H=H_{0}-p Q^{2},
\end{aligned}
$$

where $H_{0}$ - pump head with the closed position of the shutoff valves, $\mathrm{m} ; Q$ - pump flow, $\mathrm{m}^{3} / \mathrm{h}$; $p$ - hydraulic resistance of the pump, $\mathrm{m} /\left(\mathrm{m}^{3} / \mathrm{h}\right)^{2} ; \eta$ - pump efficiency.

The pump head in the closed position of the shutoff valves and its hydraulic resistance is related to the design characteristics of the pump, and the pump efficiency is a function of its supply and pressure. If we take the characteristics of the pump (6) and the characteristics of the network of pipelines (3) as identical, we obtain the equation of communication between the equation of the circulation flow and the control position of the shutoff valves:

$$
H_{0}-p Q^{2}=\left[1+h+\lambda_{\mathrm{c}} \frac{L_{\mathrm{c}}}{D}+\lambda_{\mathrm{T}} \frac{L_{\mathrm{T}}}{D}+\sum \xi_{\mathrm{c}}+\frac{2 g S^{2}}{\left(Q_{0} y\right)^{2}}\right] \frac{Q^{2} \rho}{2 S^{2}} \text {. }
$$

Using equation (7), one can calculate the magnitude of the circulation flow, having previously set the characteristics of the network of pipelines (3), the pump (6) and the position of the shutoff valves, then calculate the cooling capacity of the cooling tower using formulas (1) and (2).

Now consider an energy-efficient way to control performance with frequency regulation of the pump motor. The application of this method allows you to change the speed of the pump, and, therefore, its pressure. In this case, the initial pressure with the closed regulatory body $\mathrm{H}_{0}$ can be found by the formula [6]:

$$
\frac{H_{0}}{H_{\mathrm{A}}}=\frac{n^{2}}{n_{\mathrm{A}}^{2}}
$$

where $H_{\mathrm{A}}$ and $n$ a are the head and speed of the pump in the nominal mode.

Together, equations (7) and (8) can be expressed as the dependence of the circulation flow on the position of the shutoff valves and the number of revolutions of the pump motor, i.e. $Q=Q(y, n)$. The performance criterion of a revolving system, as a function of control parameters, can be represented by the expression $R=R(y, n)$. When calculating the revolving system, it is also necessary to take into account that with frequency control, the stop valves must be fully open, that is, $y=1$ and the water flow can be controlled only with the help of the frequency controller. The above formulas for calculations can be used in circuits with parallel connection of cooling towers and heat exchangers.

\section{Results and Discussion}

The revolving system, as a control object, is influenced by various disturbing influences. These include, for example, fluctuations in temperature and moisture content of air, changes in thermal power and the number of heat exchangers in the system. Frequency control of circulating water can be used in automatic control systems for heat exchanger parameters. During operation, it is important to know the value of the optimal flow rate of the circulating water, at which the given technological mode would be maintained. As an example, we consider a system for controlling the vacuum in the condenser of a steam turbine plant in a thermal power plant.

Using the obtained model on the basis of the frequency controller, we performed a simulation of the reverse system of the CHP. As a result, it was found that the steam load of the condenser, deposits on the heat-exchanging surfaces of the condenser, changes in hydraulic resistance, the formation of fistulas, as well as the flow of circulating water, have a significant effect on the pressure in the condenser. Therefore, a system for controlling the vacuum in the capacitor has been developed. In this system, the pressure mismatch signal in the capacitor relative to the reference is fed to the input of the controller, at the output of which a control signal is generated to the thyristor frequency converter. There is a change in the revolutions of the pump $n$ and the supply $Q$ according to relation (6), which ensures that the vacuum in the capacitor returns to its original value. At the same time, the change in the pump head is calculated by its pressure characteristics (3).

\section{Summary}

As a result, we can conclude that the circulation flow can be taken as the optimizing and controlling parameter of the cooling power of the circulating system. It is useful to use the obtained mathematical model of the circulation circuit controlled by the frequency controller of the circulation pump electric motor and/or throttling the flow by shutoff valves to solve the design and control problems of the reverse system. 


\section{Conclusion}

Practical applicability can be reflected in automated control systems when calculating the cooling capacity and specific energy costs, as well as in combination with the laws of regulation in the management of water flow. When calculating the value of the control parameter, it is taken with an error and then, using the regulation law, it is brought to the required exact value. This will improve the quality of the transition process and therefore increase production efficiency.

\section{Acknowledgments}

The work is performed according to the Russian Government Program of Competitive Growth of Kazan Federal University.

\section{References}

[1] V.A. Kuritsy. V.A. Arapov, R.L. Gorilchenko. Optimization of the process of cooling circulating water in cooling towers with the artificial draft. - Chemistry and technology of fuels and oils. 2012. No. 2.

[2] S.K. Sosnovsky, V.P. Kravchenko, Coefficient of efficiency of fan and tower cooling towers. - Heat power engineering. 2014. No 9.

[3] S.S. Berman. Heat exchangers and condensing devices of turbine units. - M.: Mashgiz. 1959.

[4] V.N. Sharifullin, A.I. Badriev, A.V. Sharifullin. Influence of irregularity of irrigation on the intensity of the process in the evaporative cooling tower. Industrial Energy, 2014, No. 6.

[5] G.G. Goppe. Reduction of energy losses in pipelines during the transportation of liquids and gases. - Energy saving and water treatment. 2008. No. 1.

[6] G.F. Bystritsky. Power equipment of industrial enterprises. - M.: IC "Academy". 2003.

[7] L.I. Kerzelli, V. Ya. Ryzhkin. Thermal power plants - M.; L.: Gosenergoizdat, 1949. - 556 p.

[8] Jiaze Ma, Chen Li, Fuyu Liu, Yufei Wang, Tiecheng Liu, Xiao Feng. Optimization of circulating cooling water networks considering the constraint of return water temperature // Journal of Cleaner Production. Volume 199, 20 October 2018, Pages 916-922.

[9] Xiaochen Zhu, Dapeng Niu, Xu Wang, Fuli Wang, Mingxing Jia. Comprehensive energy saving evaluation of circulating cooling water system based on the combination weighting method // Applied Thermal Engineering. Volume 157, 5 July 2019, 1137355 July 2019, 113735.

[10] Xiaochen Zhu, Fuli Wang, Dapeng Niu, Luping Zhao. Integrated modeling and operation optimization of circulating cooling water system based on superstructure // Applied Thermal Engineering. Volume 127, 25 December 2017, Pages 1382-1390.

[11] Tao Wu, Huimin Wei, Zhihua Ge, Lijun Yang, Xiaoze Du. Cooling water mass flow optimization for the indirect dry cooling system of thermal power unit under variable output load // International Journal of Heat and Mass Transfer. Volume 133, April 2019, Pages 1-10.

[12] Fei Song, Nan Zhang, Robin Smith, Yujiao Zeng, Jie Li, Xin Xiao. Simultaneous Optimization for Integrated Cooling Water System with Chemical Processes // Computer-Aided Chemical Engineering. Volume 43, 2018, Pages 477-482. 